\title{
Waste PET Chemical Processing to Terephthalic Amides and their Effect on Asphalt
}

\section{Performance}

Daniel R. Merkel Wenbin Kuang, Deepika Malhotra, Gayaneh Petrossian, Lirong Zhong, Kevin L. Simmons, Jinwen Zhang, Lelia Cosimbescu*

27 total figures (though some have a and b, so the number is 16)

No tables

13 pages 
NMR spectra: the chemical shifts are reported in delta $(\delta)$ units, parts per million (ppm) downfield from tetramethylsilane, which was used as an internal standard, and coupling constants are reported in Hertz $(\mathrm{Hz})$. Samples were prepared in deuterated dimethyl sulfoxide $\left(\left(\mathrm{CD}_{3}\right)_{2} \mathrm{SO}\right)$ or deuterated chloroform $\left(\mathrm{CDCl}_{3}\right)$.

Additive 1

${ }^{1} \mathrm{H}$ NMR $\left(500 \mathrm{MHz},\left(\mathrm{CD}_{3}\right)_{2} \mathrm{SO}\right) \delta 8.54(\mathrm{t}, 2 \mathrm{H}, \mathrm{J}=5.5 \mathrm{~Hz}), 7.92(\mathrm{~s}, 4 \mathrm{H}), 4.73(\mathrm{t}, 2 \mathrm{H}, \mathrm{J}=5.5 \mathrm{~Hz})$, $3.52(\mathrm{q}, 4 \mathrm{H}, \mathrm{J}=6.0 \mathrm{~Hz}), 3.33(\mathrm{q}, 4 \mathrm{H}, \mathrm{J}=6.0 \mathrm{~Hz}) .{ }^{13} \mathrm{C} \mathrm{NMR}\left(126 \mathrm{MHz},\left(\mathrm{CD}_{3}\right)_{2} \mathrm{SO}\right) \delta 165.6$, $136.6,127.1,59.7,42.2$.

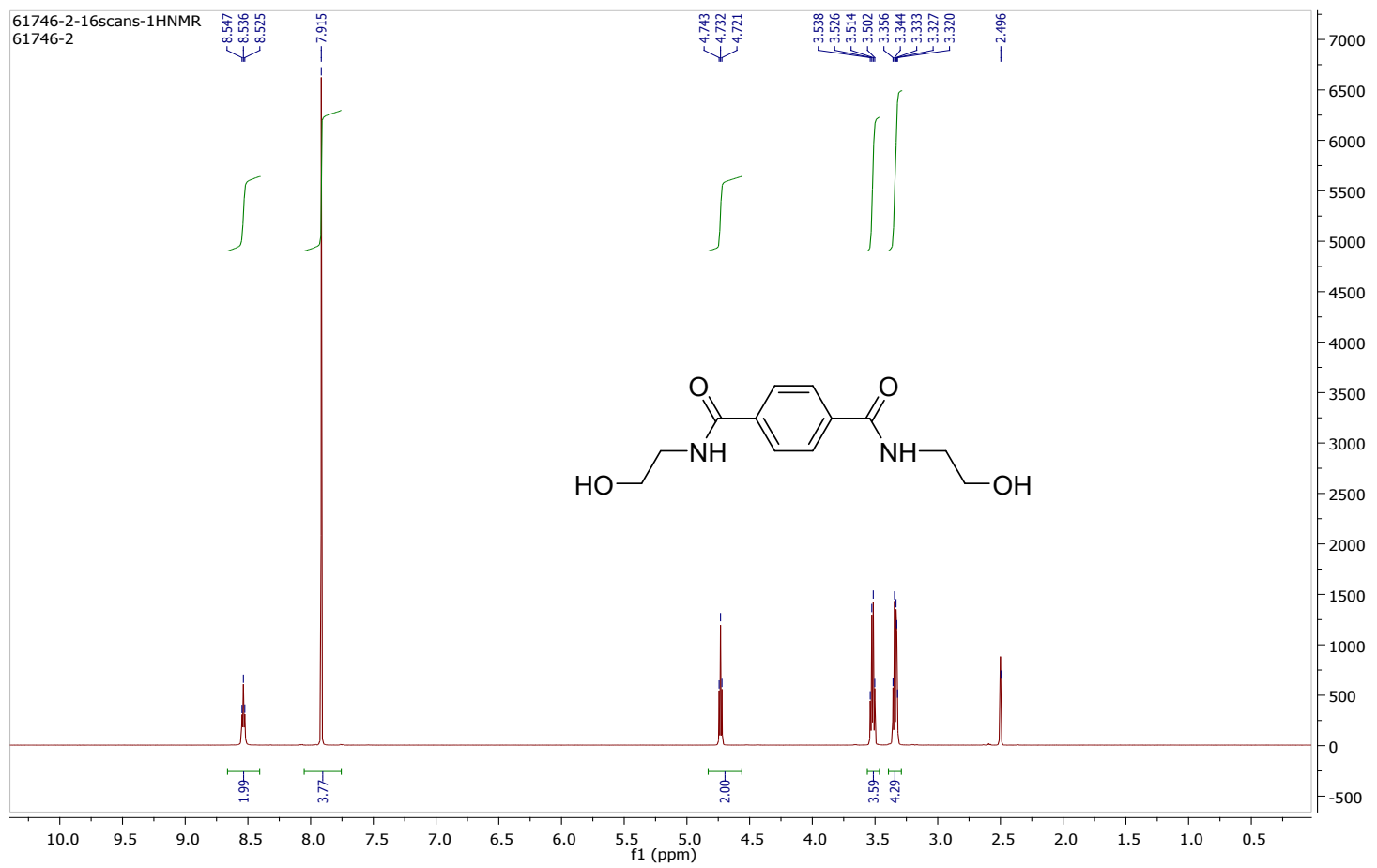

Figure S1a: ${ }^{1} \mathrm{H}$ NMR for Additive 1 


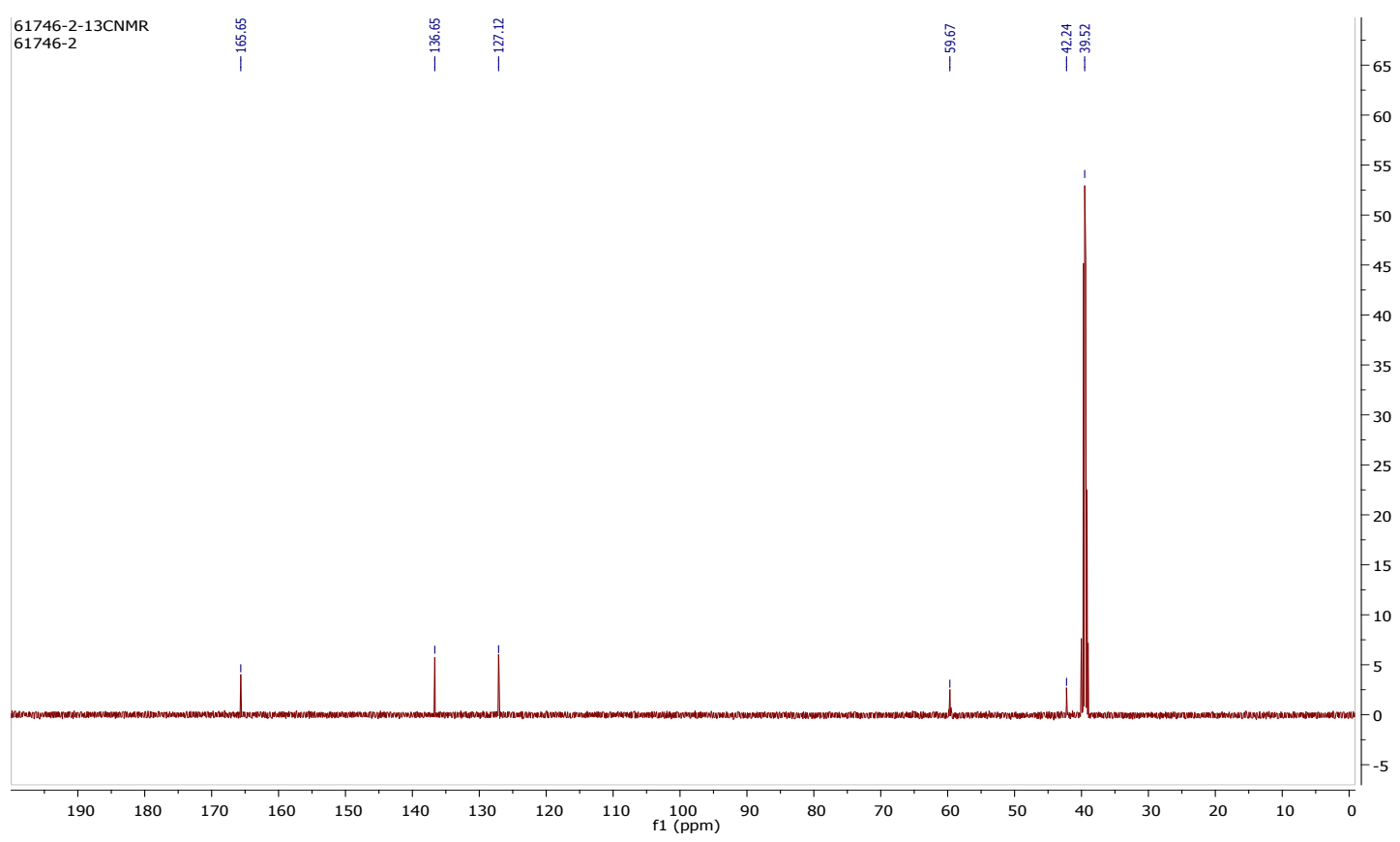

Figure S1b: ${ }^{13} \mathrm{C}$ NMR for Additive 1

Additive 2

${ }^{1} \mathrm{H} \mathrm{NMR}\left(500 \mathrm{MHz},\left(\left(\mathrm{CD}_{3}\right)_{2} \mathrm{SO}\right) \delta 8.54(\mathrm{t}, 2 \mathrm{H}, \mathrm{J}=5.5 \mathrm{~Hz}), 7.92(\mathrm{~s}, 4 \mathrm{H}), 4.73(\mathrm{t}, 2 \mathrm{H}, \mathrm{J}=5.5 \mathrm{~Hz})\right.$, $\left.3.52(\mathrm{q}, 4 \mathrm{H}, \mathrm{J}=6.0 \mathrm{~Hz}), 3.34(\mathrm{q}, 4 \mathrm{H}, \mathrm{J}=6.0 \mathrm{~Hz}) .{ }^{13} \mathrm{C} \mathrm{NMR}\left(126 \mathrm{MHz},\left(\mathrm{CD}_{3}\right)_{2} \mathrm{SO}\right)\right) \delta 165.7$, 136.7, 127.1, 59.7, 42.2.

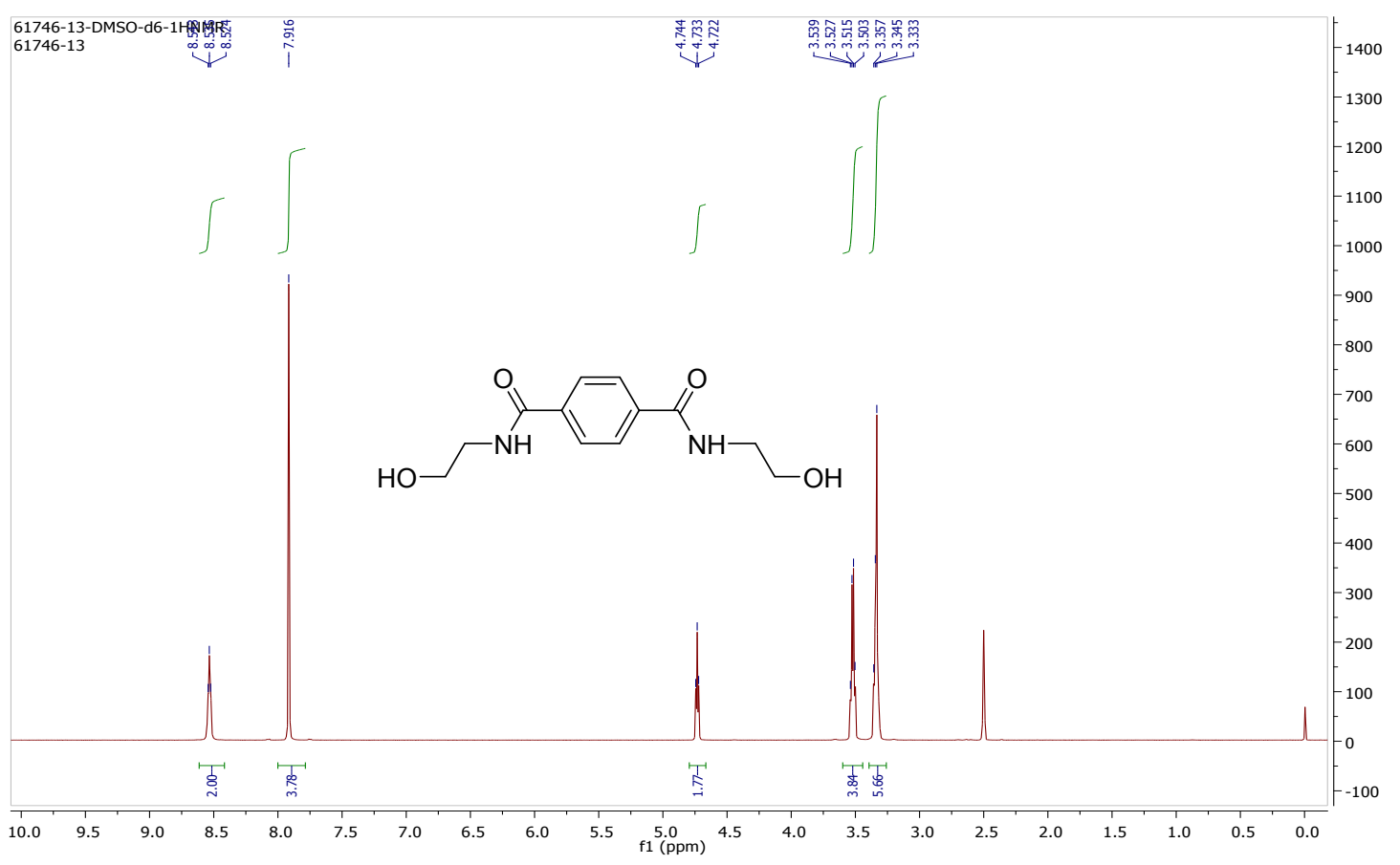

Figure S2a: ${ }^{1} \mathrm{H}$ NMR for Additive 2 


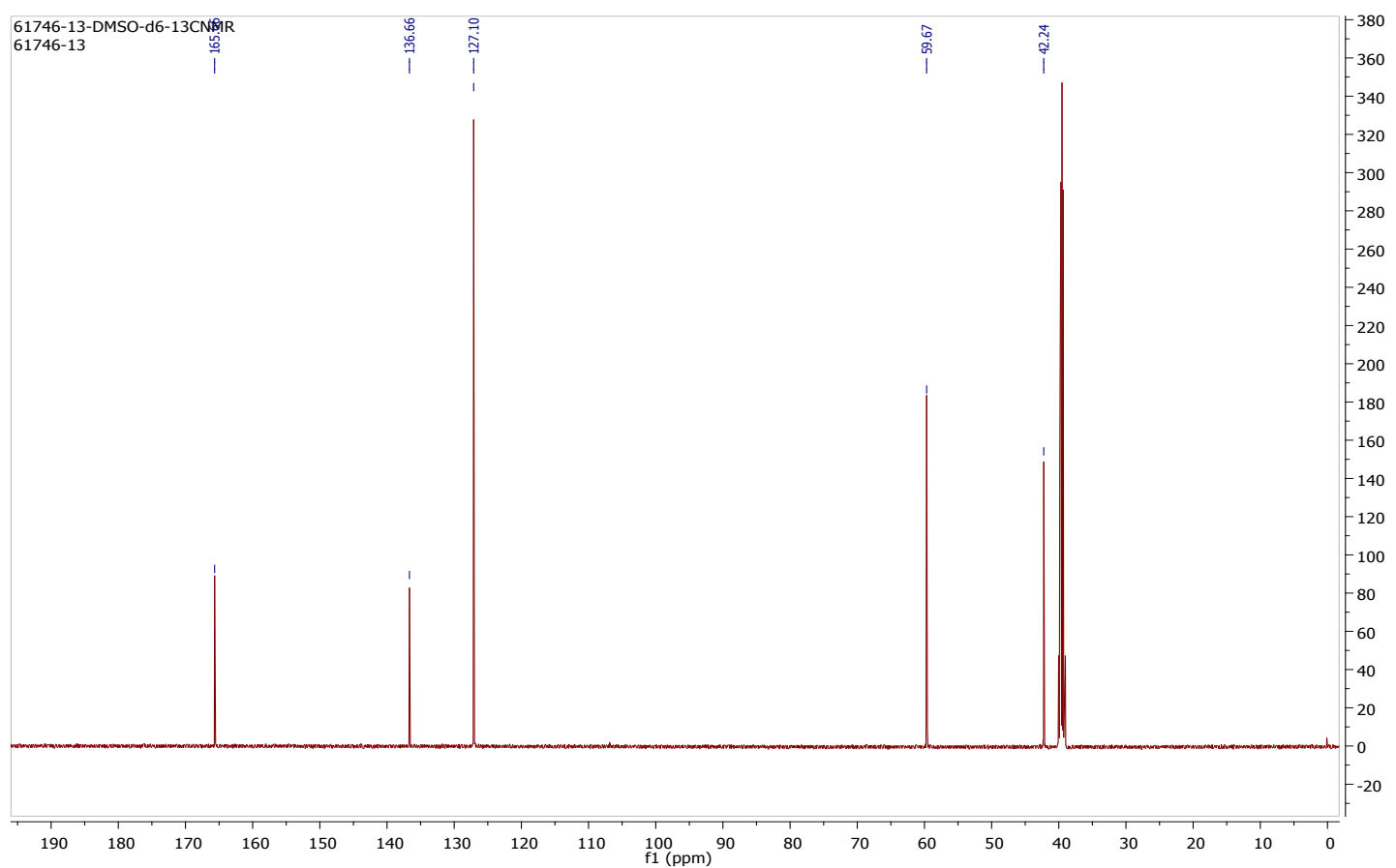

Figure S2b: ${ }^{13} \mathrm{C}$ NMR for Additive 2

Additive 3 [mixture of $\mathrm{A}(47.3 \%)$ and $\mathrm{B}(52.7 \%)$ ]

${ }^{1} \mathrm{H}$ NMR $\left(500 \mathrm{MHz}, \mathrm{CDCl}_{3}\right) \delta 8.10-8.15(4 \mathrm{H}), 7.81-7.82(4 \mathrm{H}), 6.24(0.82 \mathrm{H}), 6.15(1.47 \mathrm{H}), 4.69$ $(2 \mathrm{H}), 4.49(2 \mathrm{H}), 3.98(3 \mathrm{H}), 3.74(3 \mathrm{H}), 3.46(4 \mathrm{H}), 1.85(4 \mathrm{H}), 1.62-1.717 \mathrm{H}), 1.25(25 \mathrm{H}), 0.87$

$(6 \mathrm{H}) .{ }^{13} \mathrm{C} \mathrm{NMR}\left(126 \mathrm{MHz}, \mathrm{CDCl}_{3}\right) \delta 130.1,127.1,67.1,61.5,40.5,32.1,29.8,29.7,29.5,27.2$, 22.8, 14.3.

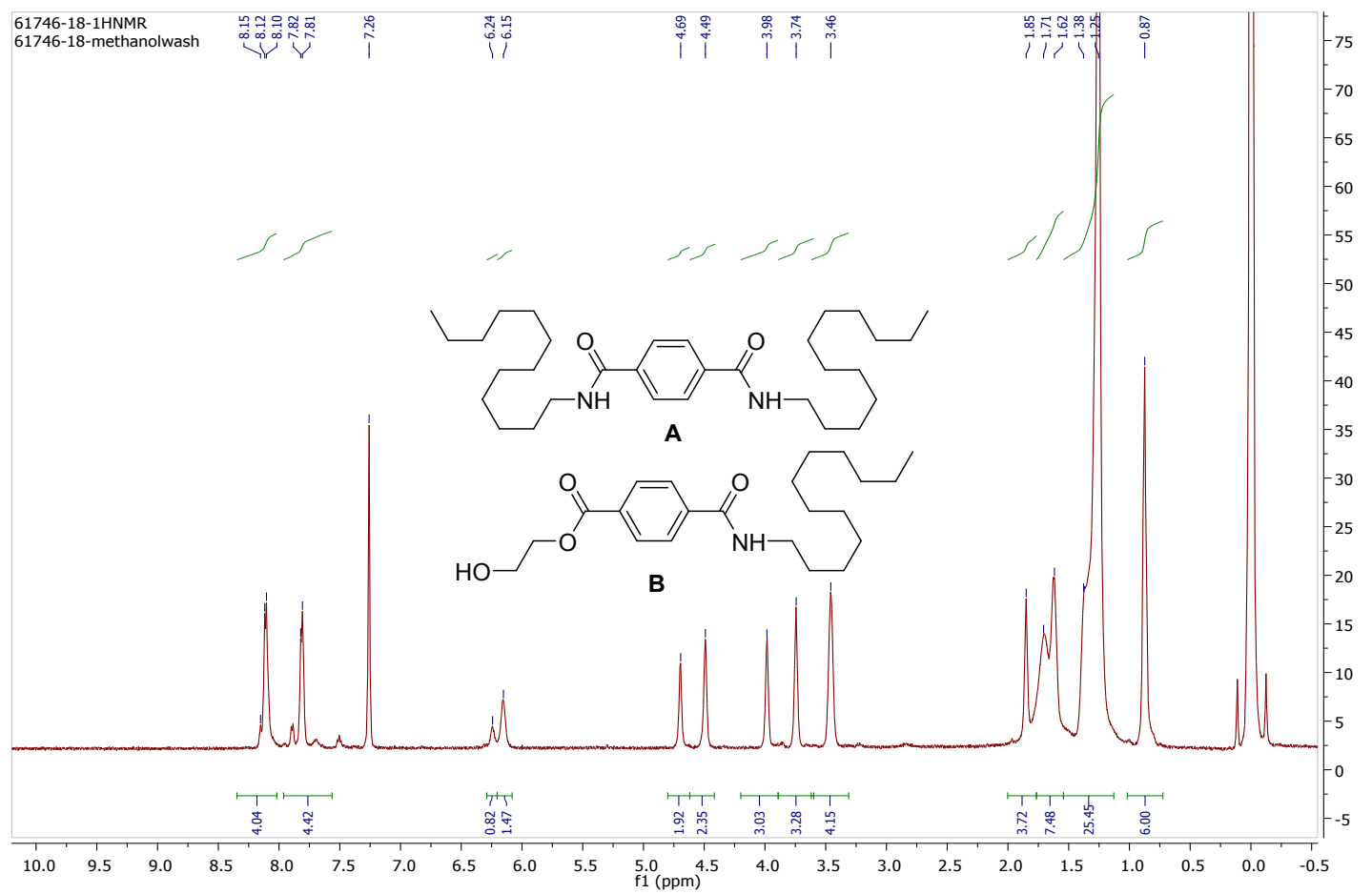


Figure S3a: ${ }^{1} \mathrm{H}$ NMR for Additive 3

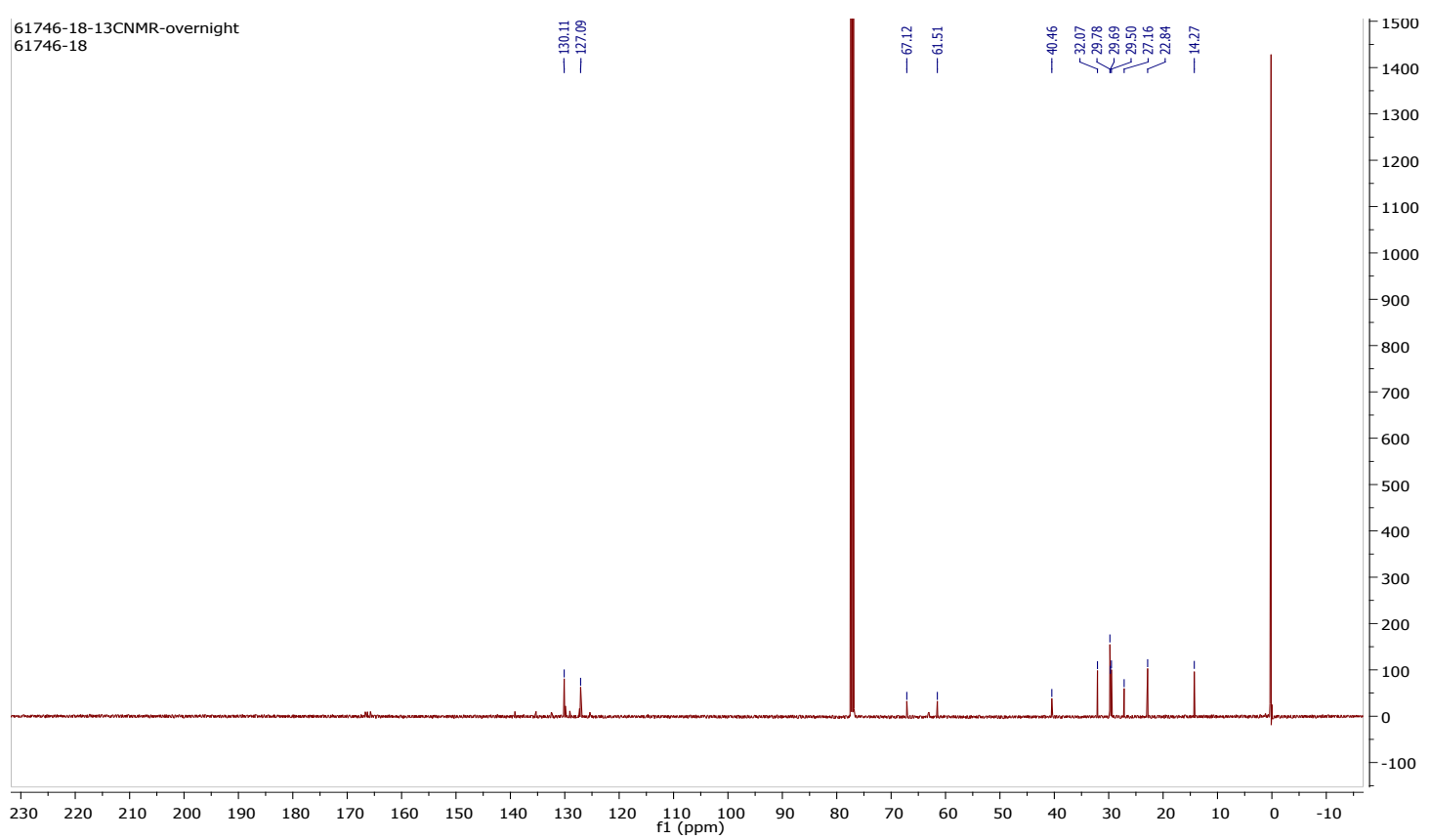

Figure S3b: ${ }^{13} \mathrm{C}$ NMR for Additive 3

Additive 4 [mixture of C (62.1\%) and D (37.9\%)]

${ }^{1} \mathrm{H}$ NMR $\left(500 \mathrm{MHz}, \mathrm{CDCl}_{3}\right) \delta 8.08-8.13(2 \mathrm{H}), 7.80(3 \mathrm{H}), 6.26(0.57 \mathrm{H}), 6.16(1.87 \mathrm{H}), 4.7(2 \mathrm{H})$, $4.49(2 \mathrm{H}), 3.98(2 \mathrm{H}), 3.74(2 \mathrm{H}), 3.44-3.48(5 \mathrm{H}), 1.85(2 \mathrm{H}), 1.61-1.64(10 \mathrm{H}), 1.32-1.40(11 \mathrm{H})$, $0.90(6 \mathrm{H}) .{ }^{13} \mathrm{C}$ NMR $\left(126 \mathrm{MHz},\left(\mathrm{CD}_{3}\right)_{2} \mathrm{SO}\right)$ and $\left(\left(\mathrm{CD}_{3}\right)_{2} \mathrm{CO}\right) \delta 139.9,137.7,77.4,70.4,50.1$, $42.0,37.1,32.9,24.0$.

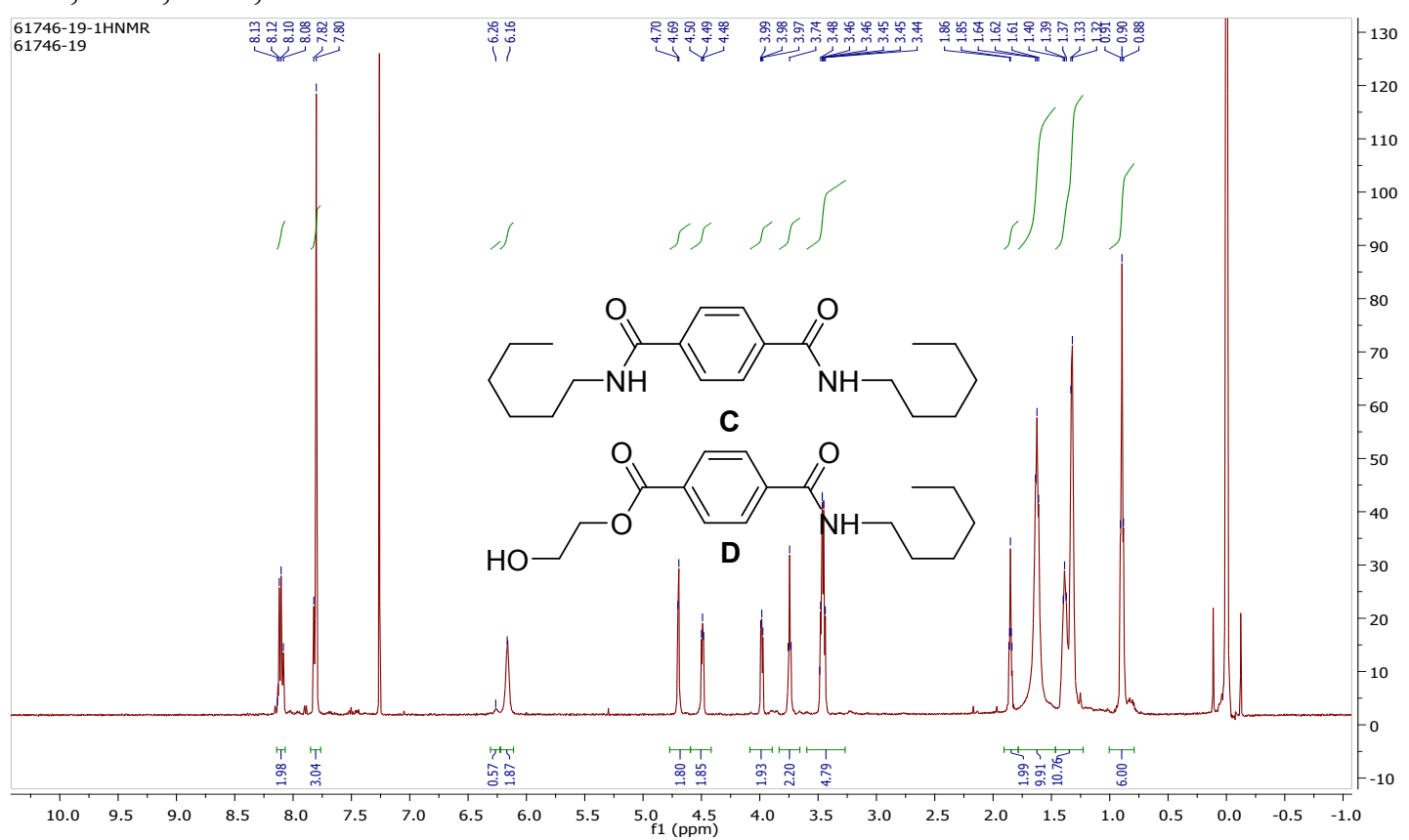

Figure S4a: ${ }^{1} \mathrm{H}$ NMR for Additive 4 


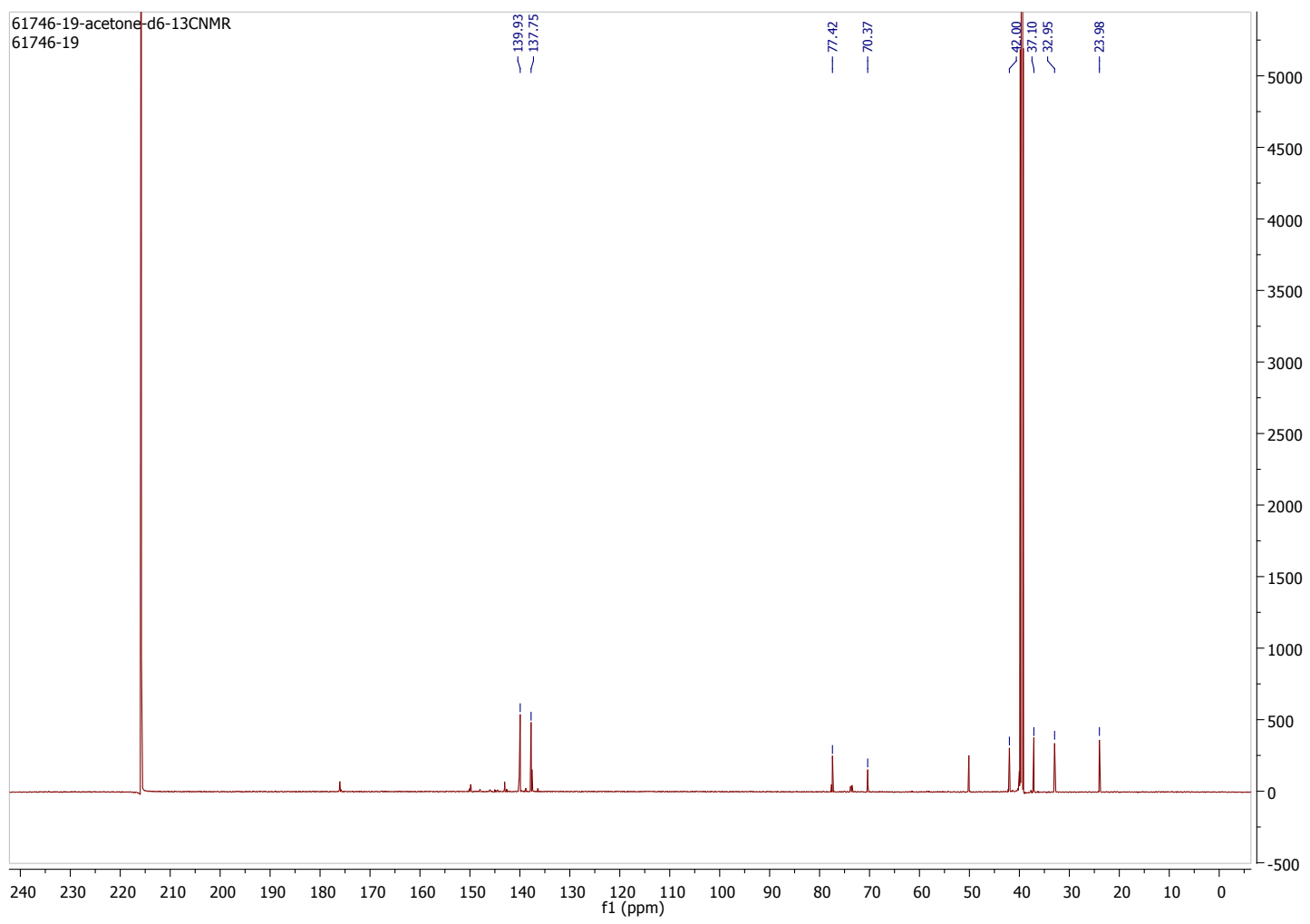

Figure S4b: ${ }^{13} \mathrm{C}$ NMR for additive 4

Additive 6

${ }^{1} \mathrm{H}$ NMR $\left(500 \mathrm{MHz},\left(\mathrm{CD}_{3}\right)_{2} \mathrm{SO}\right) \delta 8.54(\mathrm{t}, 2 \mathrm{H}, \mathrm{J}=5.5 \mathrm{~Hz}), 7.89(\mathrm{~s}, 4 \mathrm{H}), 4.47(\mathrm{t}, 2 \mathrm{H}, \mathrm{J}=5.5 \mathrm{~Hz})$, $3.46(\mathrm{q}, 4 \mathrm{H}, \mathrm{J}=6.0 \mathrm{~Hz}), 3.33(\mathrm{q}, 4 \mathrm{H}, \mathrm{J}=6.0 \mathrm{~Hz}) .{ }^{13} \mathrm{C} \mathrm{NMR}\left(126 \mathrm{MHz},\left(\mathrm{CD}_{3}\right)_{2} \mathrm{SO}\right) \delta 165.5$, $136.6,127.1,58.7,36.7,32.4$. 


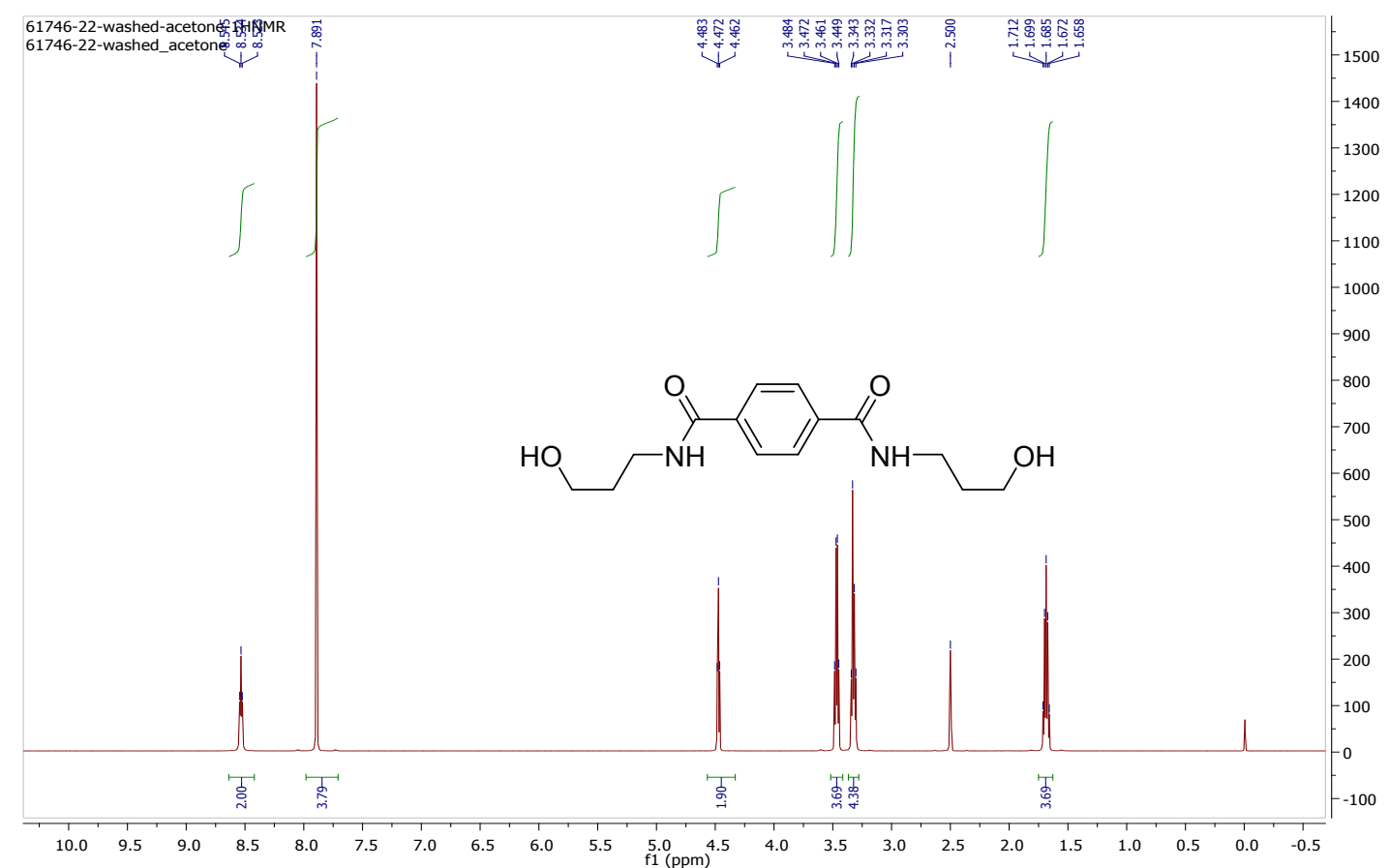

Figure S5a: ${ }^{1} \mathrm{H}$ NMR for Additive 6

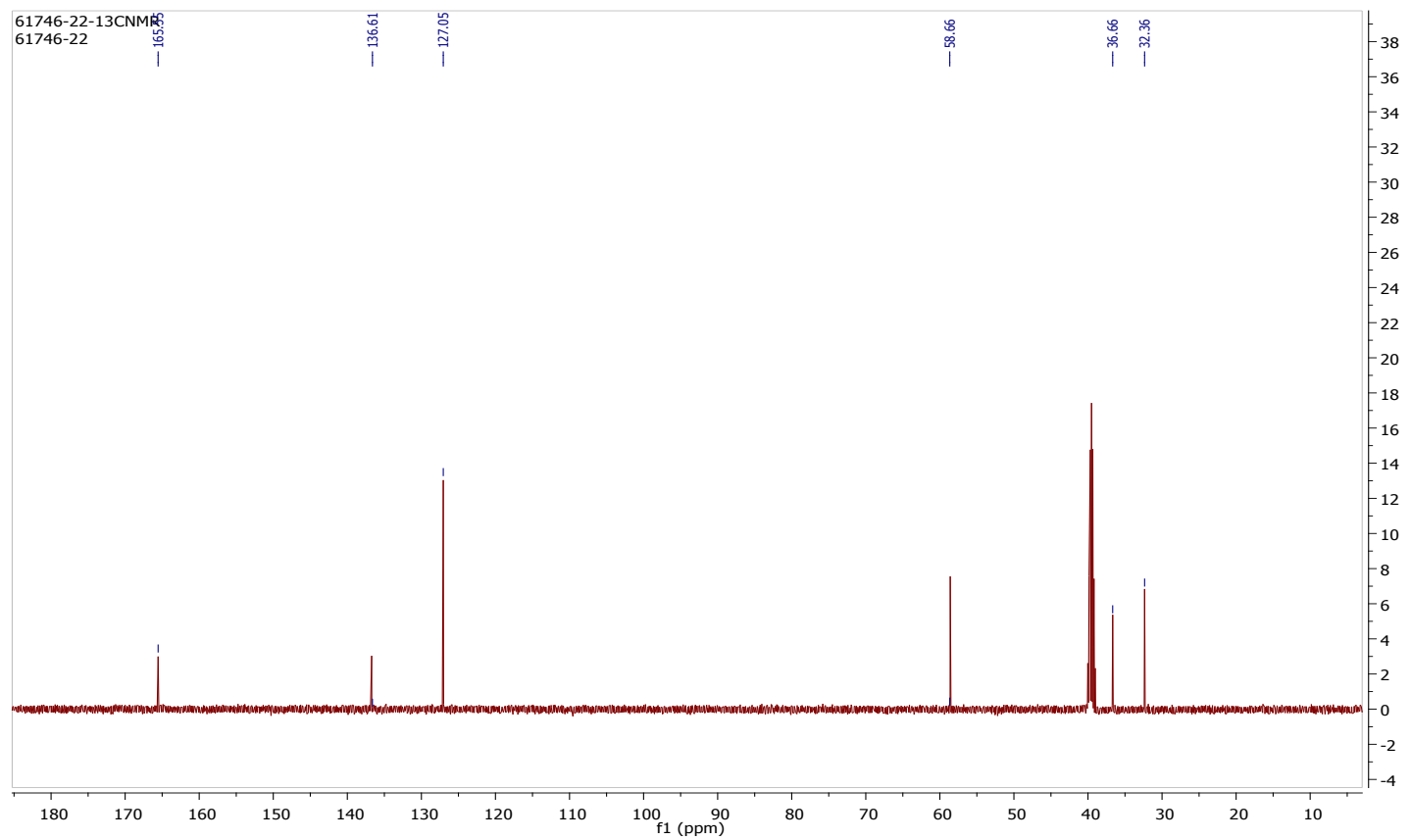

Figure S5b: ${ }^{13} \mathrm{C}$ NMR for Additive 6

Additive 7

${ }^{1} \mathrm{H}$ NMR $\left(500 \mathrm{MHz},\left(\mathrm{CD}_{3}\right)_{2} \mathrm{SO}\right) \delta 8.1(\mathrm{~d}, 2 \mathrm{H}, \mathrm{J}=8 \mathrm{~Hz}), 7.92(\mathrm{~s}, 4 \mathrm{H}), 4.67(\mathrm{t}, 2 \mathrm{H}, \mathrm{J}=5.0 \mathrm{~Hz})$, 3.84-3.90 (m, 2H), 3.45-3.49 (m, 2H), 3.39-3.42 (m, 2H), 1.62-1.70 (m, 2H), 1.41-1.50 (m, 2H), $0.87(\mathrm{t}, 6 \mathrm{H}, \mathrm{J}=7.5 \mathrm{~Hz}) .{ }^{13} \mathrm{C}$ NMR $\left(126 \mathrm{MHz},\left(\mathrm{CD}_{3}\right)_{2} \mathrm{SO}\right) \delta 165.6,136.9,127.2,63.0,53.2,23.7$, 10.7 . 


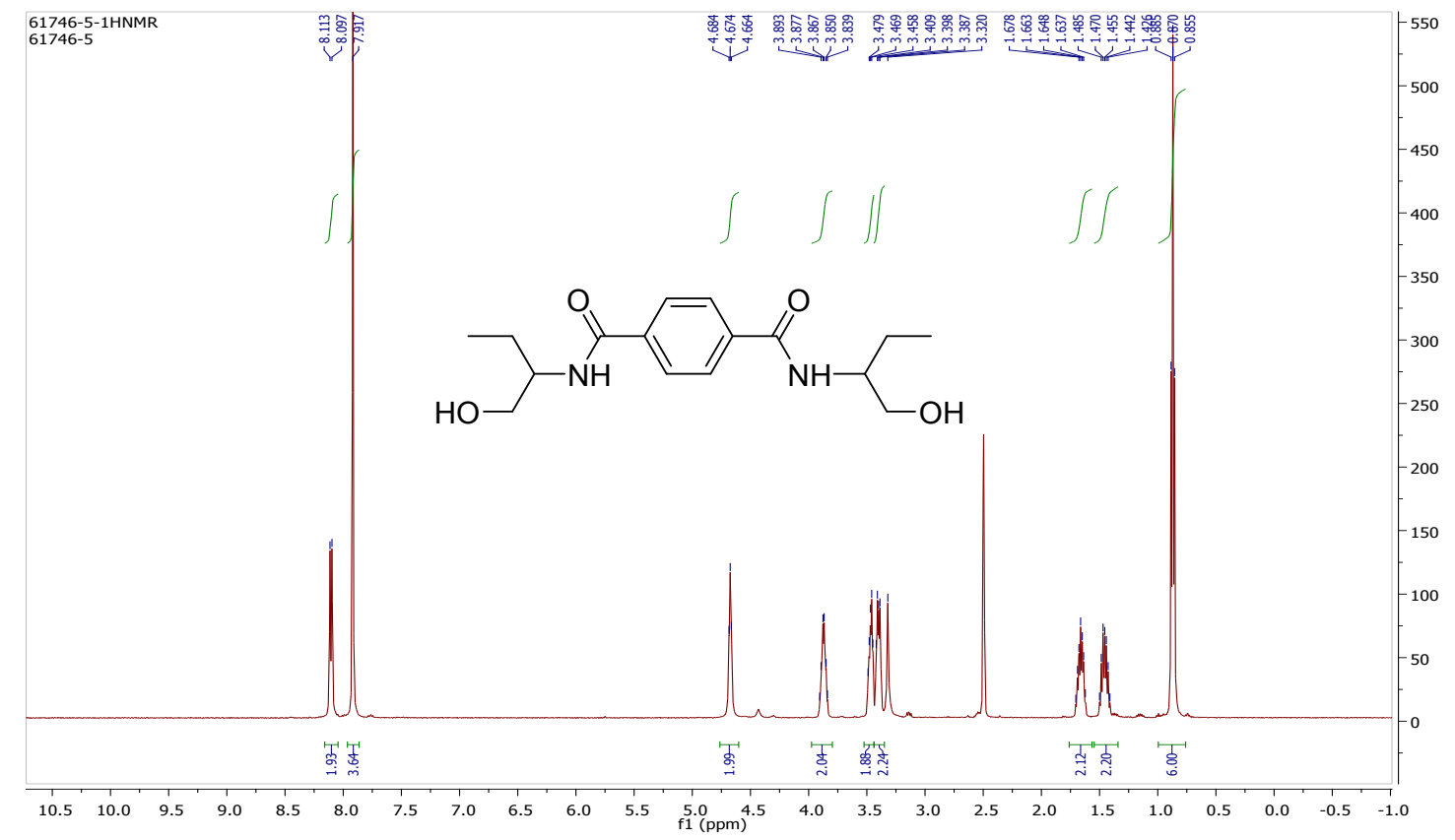

Figure S6a: ${ }^{1} \mathrm{H}$ NMR for Additive 7

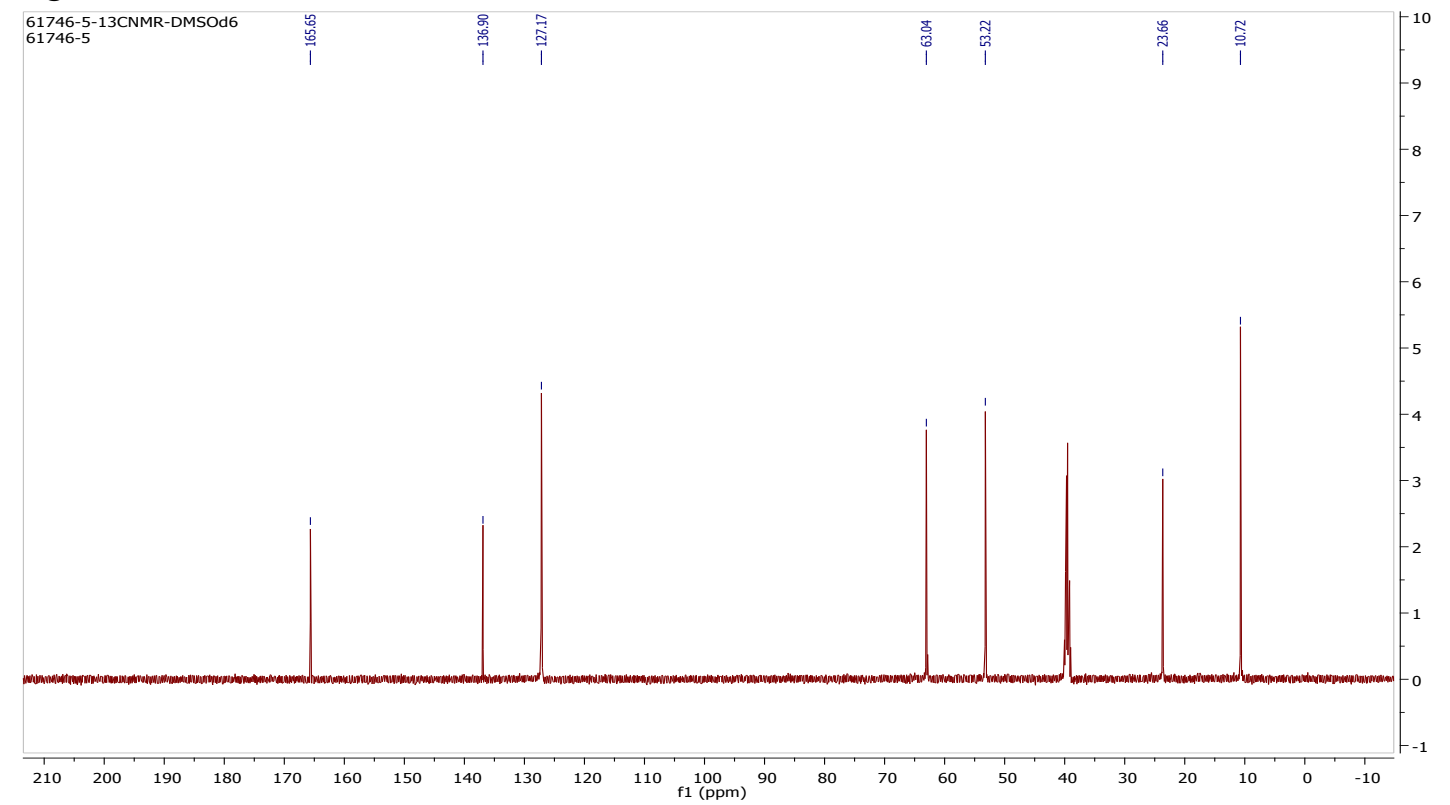

Figure S6b: ${ }^{13} \mathrm{C}$ NMR for Additive 7

Additive 8

${ }^{1} \mathrm{H}$ NMR (500 MHz, $\left.\left(\mathrm{CD}_{3}\right)_{2} \mathrm{SO}\right) 8.54$ (t, 2H, J = $\left.5.0 \mathrm{~Hz}\right), 7.89(\mathrm{~s}, 4 \mathrm{H}), 4.28(\mathrm{bs}, 2 \mathrm{H}), 3.39$ (m, $4 \mathrm{H}), 3.21-3.28(\mathrm{~m}, 4 \mathrm{H}), 1.28-1.55(\mathrm{~m}, 12 \mathrm{H}) .{ }^{13} \mathrm{C}$ NMR $\left(126 \mathrm{MHz},\left(\mathrm{CD}_{3}\right)_{2} \mathrm{SO}\right) \delta 165.4,136.8$, $127.1,60.6,32.3,29.0,23.1$. 


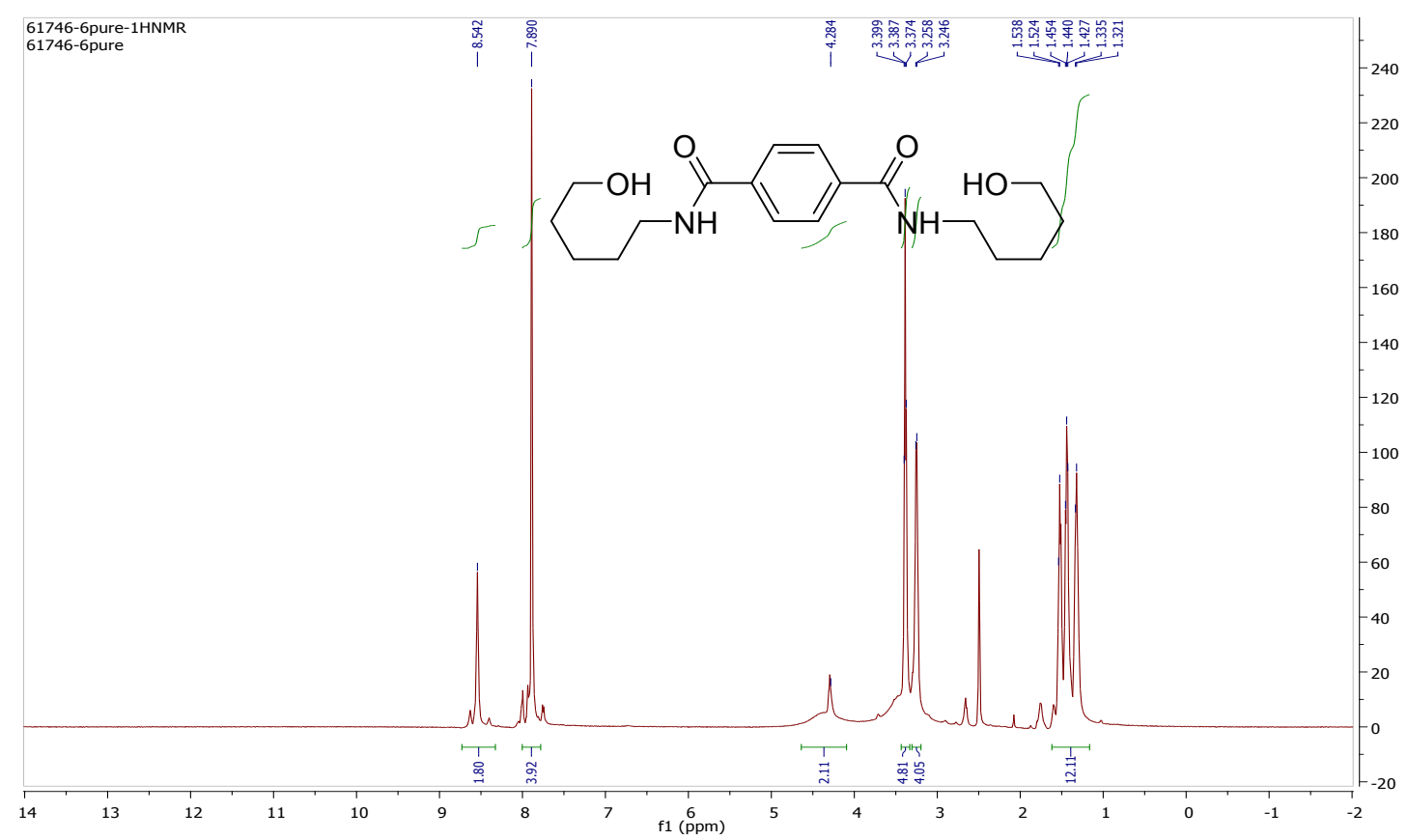

Figure S7a: ${ }^{1} \mathrm{H}$ NMR for Additive 8

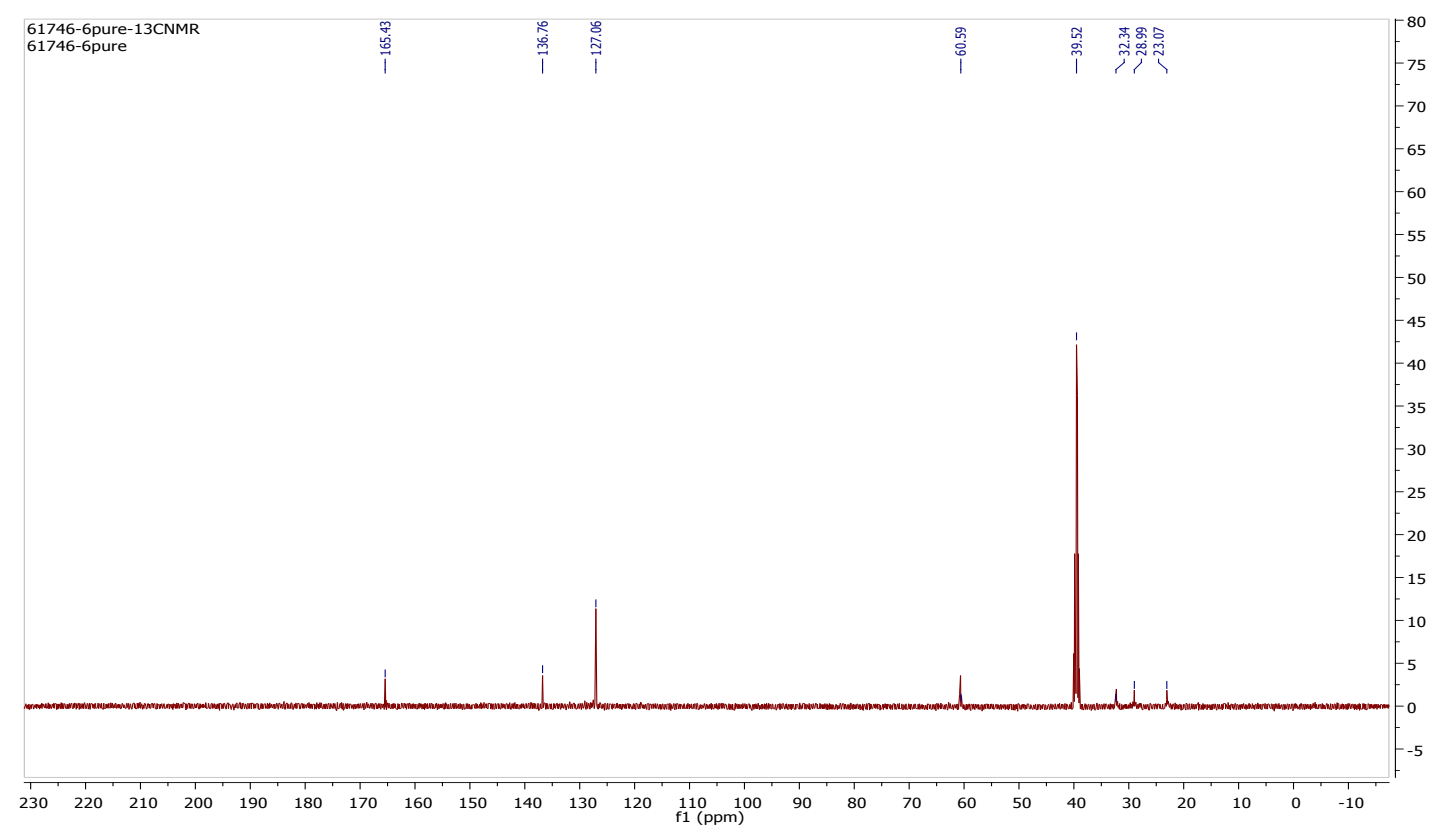

Figure S7b: ${ }^{13} \mathrm{C}$ MR for Additive 8

Additive 9

${ }^{1} \mathrm{H}$ NMR (500 MHz, $\left.\mathrm{CDCl}_{3}\right) \delta 8.09-8.12(2 \mathrm{H}, \mathrm{m}), 7.81(3 \mathrm{H}, \mathrm{s}), 6.11(2 \mathrm{H}), 4.70(1 \mathrm{H}), 3.75(4 \mathrm{H})$, $3.45(2 \mathrm{H}), 1.86(5 \mathrm{H}), 1.59-1.66(4 \mathrm{H}), 1.56(12 \mathrm{H}), 1.26-1.41(14 \mathrm{H}), 0.88(6 \mathrm{H})$. 


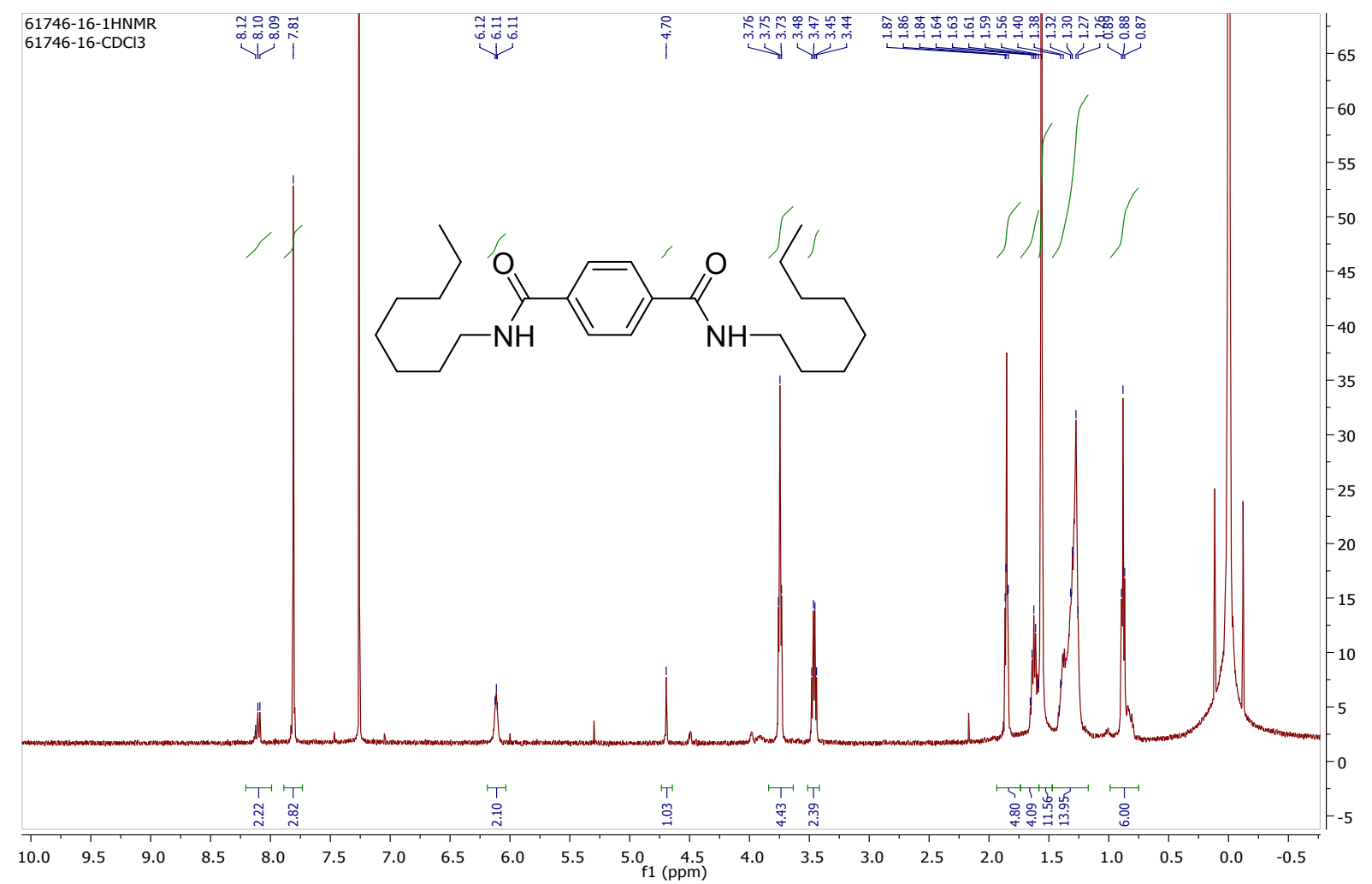

Figure S8a: ${ }^{1} \mathrm{H}$ NMR for Additive 9

\section{Differential Scanning Calorimetry}

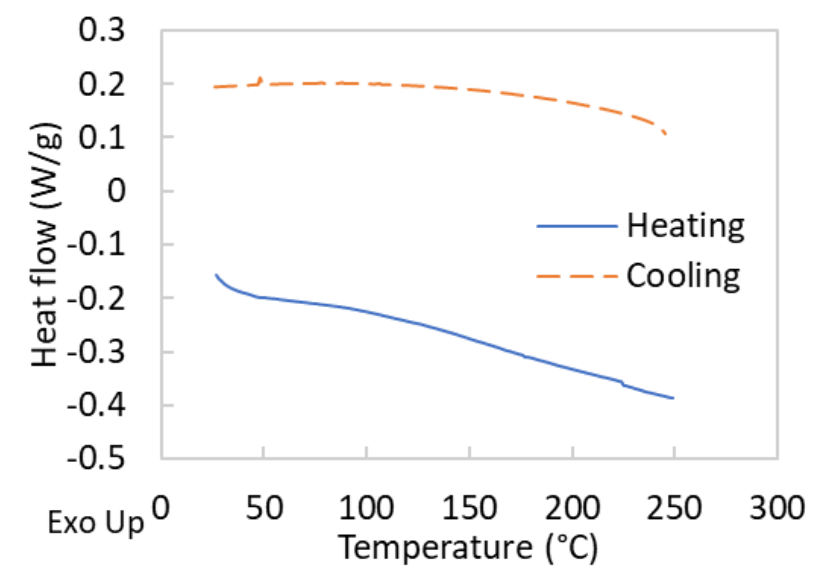

Figure S9. DSC heating and cooling thermographs of neat asphalt. 

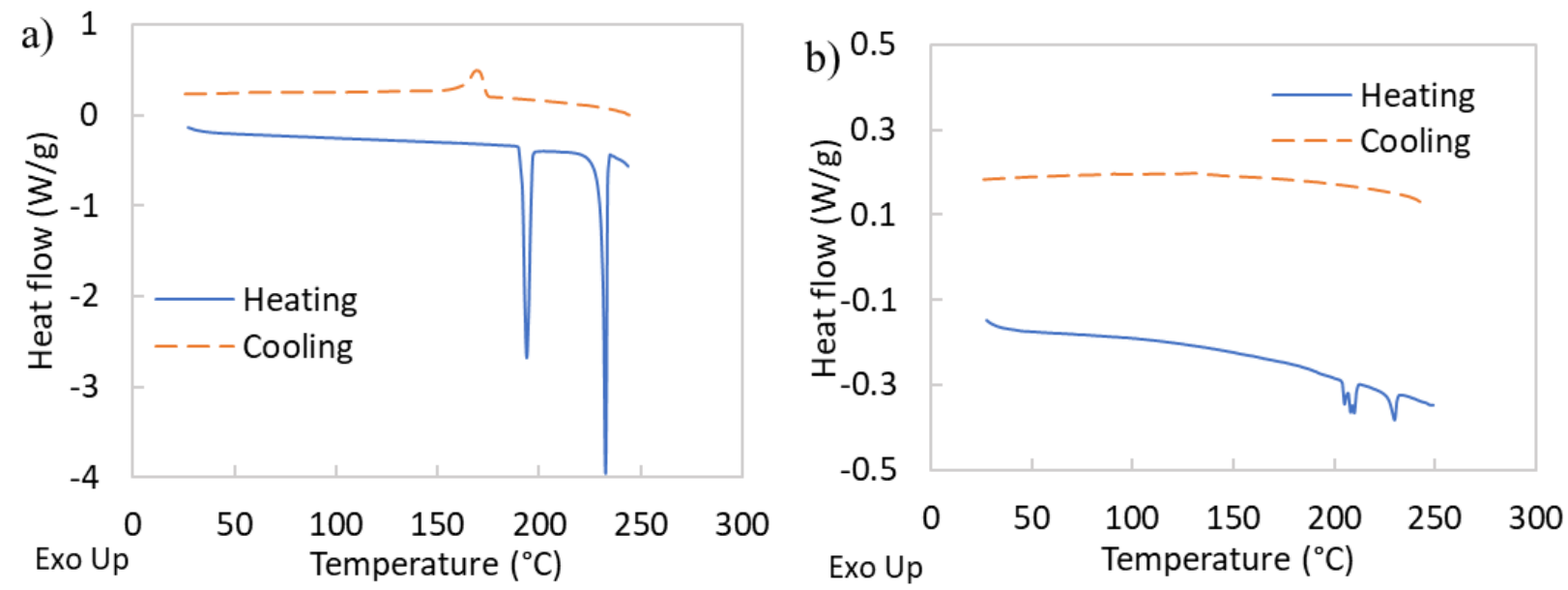

Figure S10. DSC heating and cooling thermographs of (a) additive $1\left(\mathrm{~N}^{1}, \mathrm{~N}^{4}-\mathrm{bis}(2-\right.$ hydroxyethyl)terephthalamide from clean PET source) and (b) asphalt with $5 \mathrm{wt} \%$ additive 1.
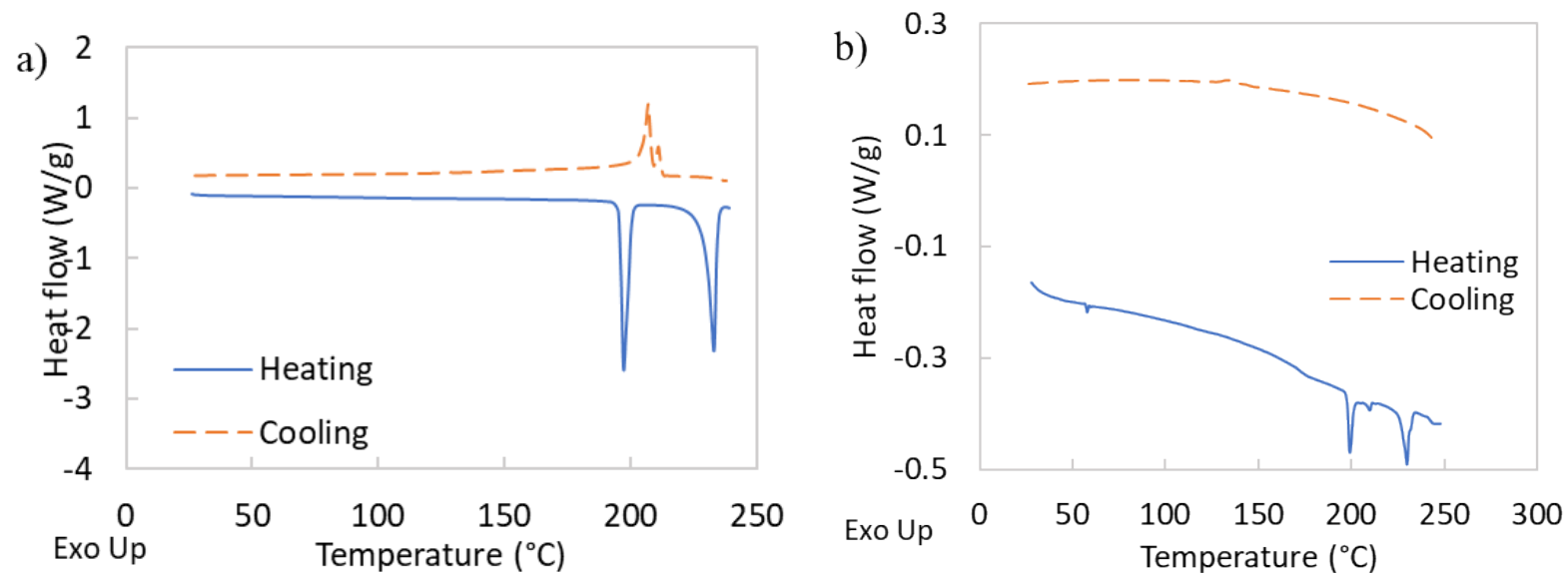

Figure S11: DSC heating and cooling thermographs of (a) additive $2\left(\mathrm{~N}^{1}, \mathrm{~N}^{4}-\right.$ bis $(2-$ hydroxyethyl)terephthalamide from mixed-waste PET source) and (b) asphalt with $5 \mathrm{wt} \%$ additive 2 .
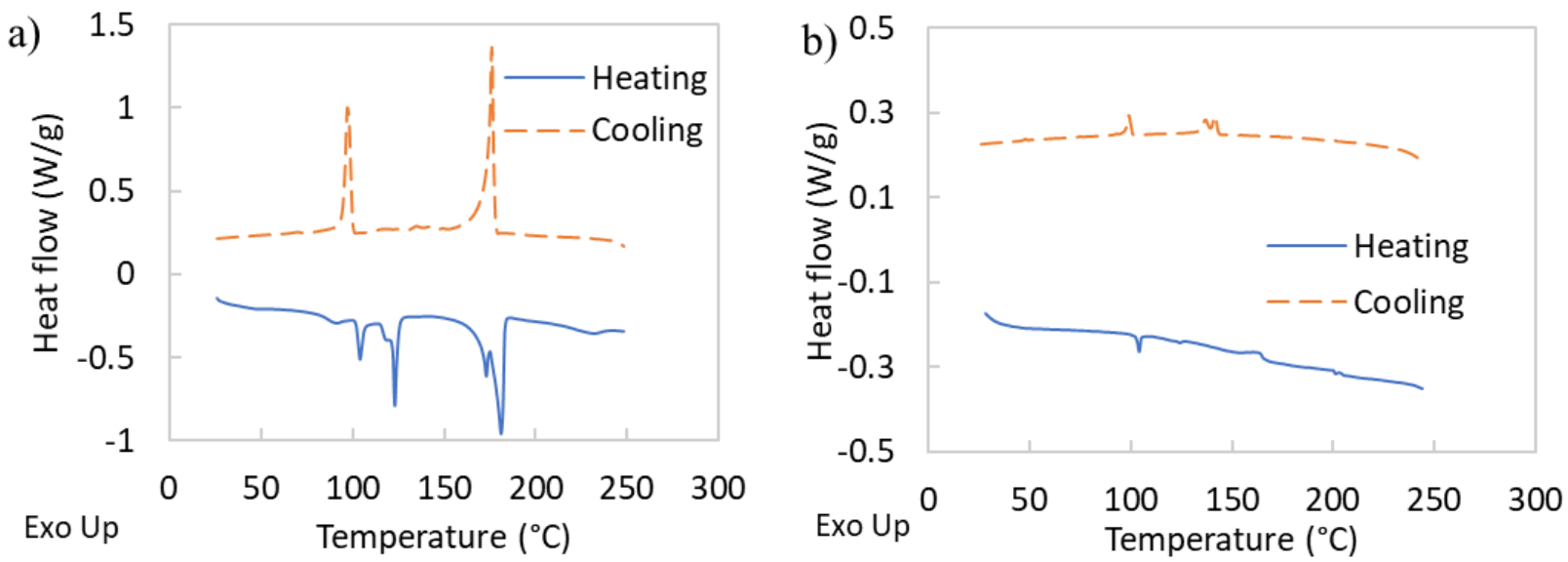

Figure S12. DSC heating and cooling thermographs of (a) additive $3\left(\mathrm{~N}^{1}, \mathrm{~N}^{4}-\right.$ didodecylterephthalamide) and (b) asphalt with $5 \mathrm{wt} \%$ additive 3 . 

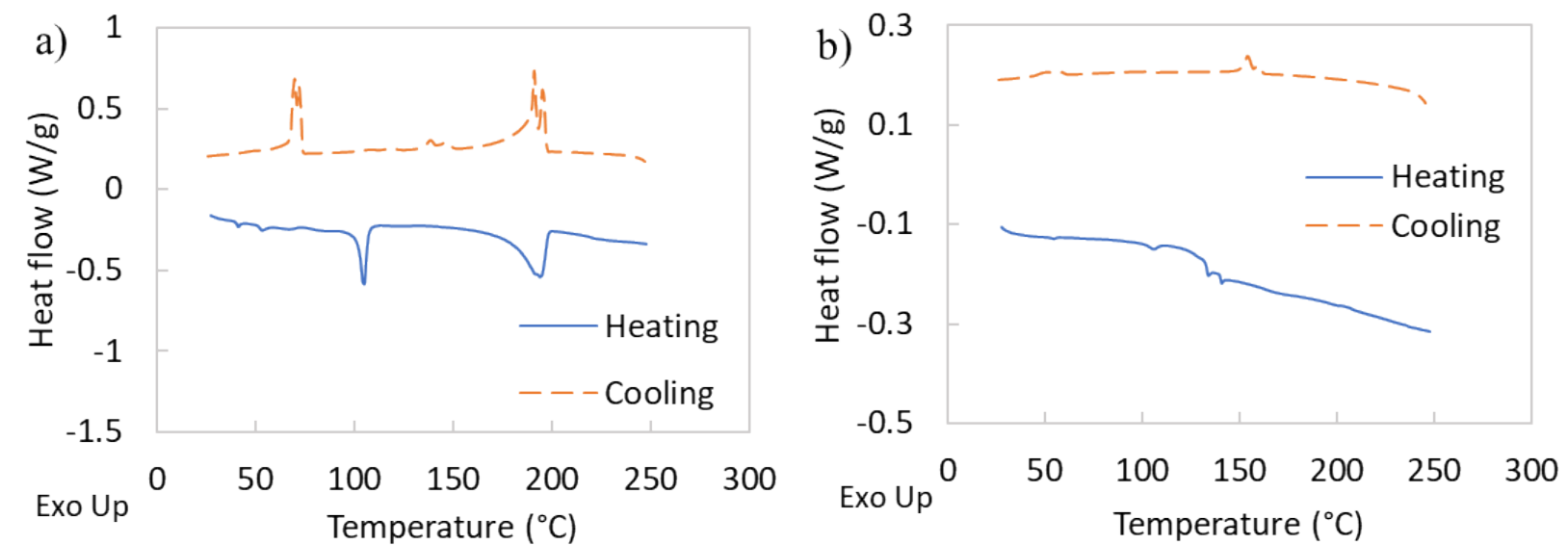

Figure S13. DSC heating and cooling thermographs of (a) additive $4\left(\mathrm{~N}^{1}, \mathrm{~N}^{4}\right.$ dihexylterephthalamide) and (b) asphalt with $5 \mathrm{wt} \%$ additive 4.

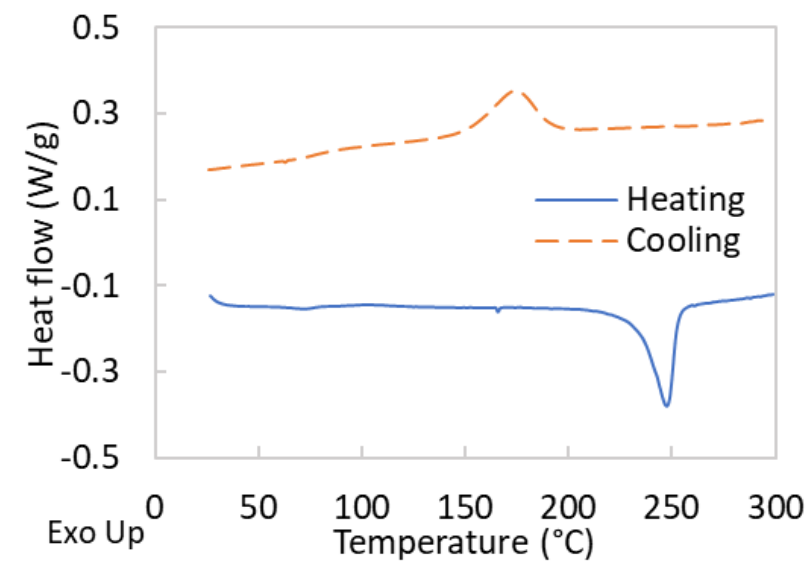

Figure S14. DSC heating and cooling thermographs of neat PET (additive 5).

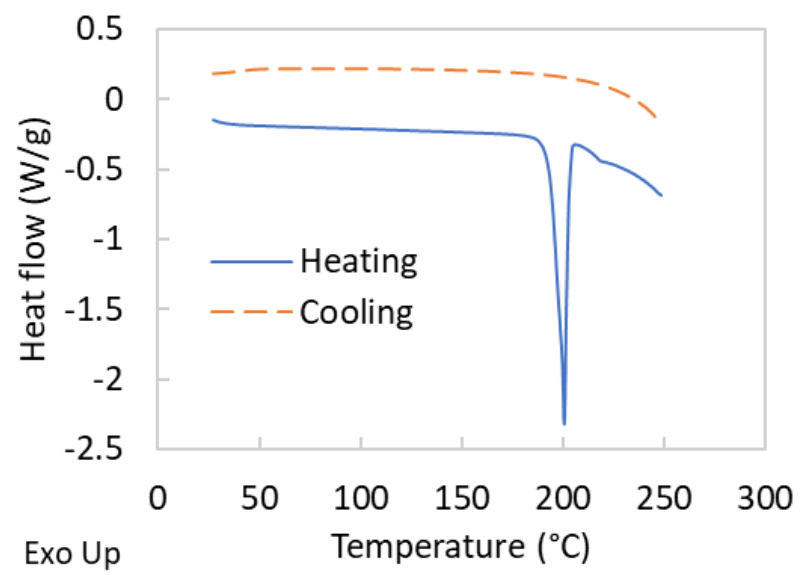

Figure S15. DSC heating and cooling thermographs of additive $7\left(\mathrm{~N}^{1}, \mathrm{~N}^{4}\right.$-diethyl- $\mathrm{N}^{1}, \mathrm{~N}^{4}-$ bis $(2-$ hydroxyethyl)terephthalamide). 


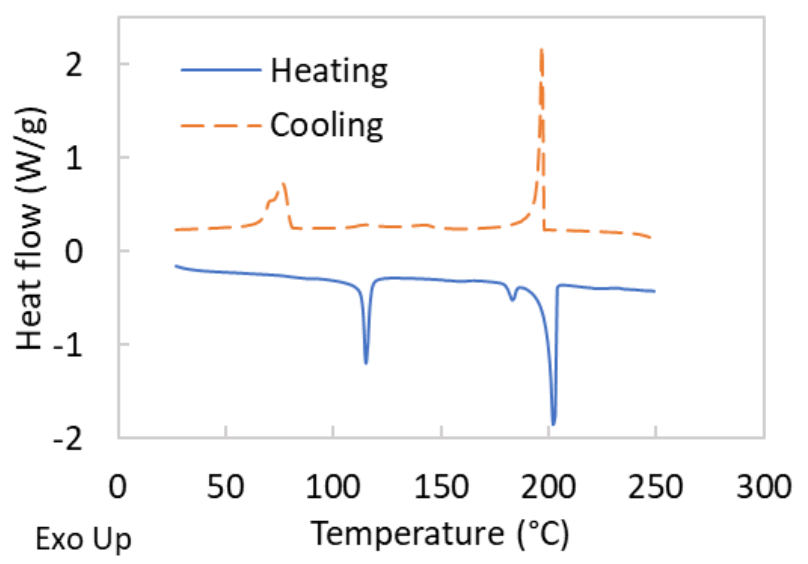

Figure S16. DSC heating and cooling thermographs of additive $9\left(\mathrm{~N}^{1}, \mathrm{~N}^{4}-\right.$ dioctylterephthalamide). 